\title{
AT Cnc: A SECOND DWARF NOVA WITH A CLASSICAL NOVA SHELL
}

\author{
Michael M. Shara ${ }^{1,5}$, Trisha Mizusawa ${ }^{1}$, Peter Wehinger ${ }^{2}$, David Zurek ${ }^{1,5,6}$, \\ Christopher D. Martin ${ }^{3}$, James D. Neill $^{3}$, Karl Forster $^{3}$, and Mark Seibert ${ }^{4}$ \\ ${ }^{1}$ Department of Astrophysics, American Museum of Natural History, Central Park West at 79th Street, New York, NY 10024-5192, USA \\ ${ }^{2}$ Steward Observatory, the University of Arizona, 933 North Cherry Avenue, Tucson, AZ 85721, USA \\ ${ }^{3}$ Department of Physics, Math and Astronomy, California Institute of Technology, 1200 East California Boulevard, Mail Code 405-47, Pasadena, CA 91125, USA \\ ${ }^{4}$ Observatories of the Carnegie Institution of Washington, 813 Santa Barbara Street, Pasadena, CA 91101, USA \\ Received 2012 July 31; accepted 2012 August 23; published 2012 October 8
}

\begin{abstract}
We are systematically surveying all known and suspected Z Cam-type dwarf novae for classical nova shells. This survey is motivated by the discovery of the largest known classical nova shell, which surrounds the archetypal dwarf nova Z Camelopardalis. The Z Cam shell demonstrates that at least some dwarf novae must have undergone classical nova eruptions in the past, and that at least some classical novae become dwarf novae long after their nova thermonuclear outbursts, in accord with the hibernation scenario of cataclysmic binaries. Here we report the detection of a fragmented "shell," 3 arcmin in diameter, surrounding the dwarf nova AT Cancri. This second discovery demonstrates that nova shells surrounding Z Cam-type dwarf novae cannot be very rare. The shell geometry is suggestive of bipolar, conical ejection seen nearly pole-on. A spectrum of the brightest AT Cnc shell knot is similar to that of the ejecta of the classical nova GK Per, and of Z Cam, dominated by [N II] emission. Galaxy Evolution Explorer FUV imagery reveals a similar-sized, FUV-emitting shell. We determine a distance of $460 \mathrm{pc}$ to AT Cnc, and an upper limit to its ejecta mass of $\sim 5 \times 10^{-5} M_{\odot}$, typical of classical novae.
\end{abstract}

Key words: novae, cataclysmic variables - stars: individual (AT Cancri)

\section{INTRODUCTION AND MOTIVATION}

Dwarf and classical novae are all close binary stars, wherein a white dwarf accretes hydrogen-rich matter from its Roche lobe filling companion, or from the wind of a nearby giant. In dwarf novae, an instability (Osaki 1974) episodically dumps much of the accretion disk onto the white dwarf. The liberation of gravitational potential energy then brightens these systems by up to 100-fold every few weeks or months (Warner 1995). This accretion process in dwarf novae must inevitably build an electron degenerate, hydrogen-rich envelope on the white dwarf (Shara et al. 1986). Theory and detailed simulations predict that once the accreted mass $M_{\text {env }}$ reaches of the order of $10^{-5} M_{\odot}$, a thermonuclear runaway (TNR) will occur in the degenerate layer of accreted hydrogen. The TNR causes the rapid rise to $\sim 10^{5} L_{\odot}$ or more, and the high-speed ejection of the accreted envelope (Shara 1989; Yaron et al. 2005) in a classical nova explosion. Theory thus predicts that dwarf novae must inevitably give rise to classical novae.

Collazzi et al. (2009) have updated the seminal work of Robinson (1975), finding no evidence for dwarf nova eruptions in the progenitors of classical novae during the decades before the nova explosions. The identified progenitors of almost all classical novae are instead nova-like variables, in which the mass transfer rate $\dot{M}$ through the accretion disk is too high to permit the disk instability that drives dwarf nova eruptions. This apparent contradiction with theory is explained by the hibernation scenario of cataclysmic variables (Shara et al. 1986) as follows.

During the millennia before a nova eruption, gravitational radiation drives the white and red dwarfs closer together,

\footnotetext{
5 Visiting Astronomer, Kitt Peak National Observatory, National Optical Astronomy Observatory, which is operated by the Association of Universities for Research in Astronomy (AURA) under cooperative agreement with the National Science Foundation.

6 Visiting Astronomer, Steward Observatory, the University of Arizona, 933 North Cherry Avenue, Tucson, AZ 85721, USA.
}

enhancing Roche lobe overflow and $\dot{M}$. The increasingly high mass transfer rate turns a dwarf nova into a nova-like variable centuries before the envelope mass reaches the value needed for a TNR. The higher $\dot{M}$ of a nova-like variable chokes off dwarf nova eruptions, hence none are seen as nova progenitors. During the few centuries after a nova eruption the mass transfer rate remains high (due to irradiation of the red dwarf), which again prevents dwarf nova eruptions. A few centuries after a nova eruption, the hibernation scenario predicts that dwarf nova eruptions should begin anew. This is because irradiation of the red dwarf by the cooling white dwarf drops, as does $\dot{M}$. These newly reborn dwarf nova will be the highest mass transfer rate dwarf novae- the Z Camelopardalis stars. Thus, within the context of the hibernation scenario, one expects old novae to evolve from nova-like variables into Z Cam stars in the centuries after nova eruptions. Only these Z Cam stars will be surrounded by old nova shells. As gravitational radiation eventually drives the two stars in a CV together, one expects $\mathrm{Z}$ Cam stars to be the most likely progenitors of the nova-like variables before they erupt as classical novae. These Z Cam stars will not be surrounded by old nova shells- - their shells dispersed many millennia ago. The hibernation scenario thus predicts that some, but not all Z Cam stars should be surrounded by old nova shells.

In 2007 we reported the discovery of a classical nova shell surrounding the prototypical dwarf nova $\mathrm{Z}$ Camelopardalis (Shara et al. 2007). This shell is an order of magnitude more extended than those detected around any other classical nova. The derived shell mass matches that of classical novae, and is inconsistent with the mass expected from a dwarf nova wind or a planetary nebula. The Z Cam shell observationally linked, for the first time, a prototypical dwarf nova with an ancient nova eruption and the classical nova process. This was the firstever confirmation of a key prediction of cataclysmic binary TNR theory: the accreting white dwarfs in dwarf novae must eventually erupt as classical novae. 
Motivated by this discovery, we have been searching for other nova shells surrounding dwarf novae. One of our targets was the Z Cam-like dwarf nova AT Cancri. In a study of AT Cnc, Bond \& Tifft (1974) found shallow, broad absorption lines, and suggested that the star is an eclipsing binary composed of a DA white dwarf and a faint red dwarf companion. Nogami et al. (1999) found the orbital period to be 0.2011 days, and detected $\mathrm{P}$ Cygni profiles in the asymmetric $\mathrm{H} \alpha$ line. A summary of AT Cnc's properties, as well as spectroscopic and photometric observations, are given by Nogami et al. (1999).

Our optical narrowband imaging of AT Cnc immediately revealed fragmented rings surrounding the star. Follow-up observations with Galaxy Evolution Explorer (GALEX) confirmed the presence of FUV-emitting material surrounding this dwarf nova.

In Section 2, we describe our observations. We show optical narrowband imagery of the rings of material surrounding AT Cancri in Section 3, and a spectrum of the shell material in Section 4. GALEX ultraviolet imagery is presented in Section 5. We determine the distance to AT Cnc, and an upper limit to its ejecta mass in Section 6. The age of the AT Cnc ejecta is discussed in Section 7. The implications of the existence of the ejecta are considered in Section 8, and we briefly summarize our results in Section 9.

\section{OBSERVATIONS AND IMAGE PROCESSING}

Narrowband images of AT Cnc in the lines of $\mathrm{H} \alpha$ and [N II], and broadband $R$ images were obtained with the 90Prime camera (Williams et al. 2004) of the $2.3 \mathrm{~m}$ Steward Observatory telescope on 2007 November 11. The camera's focal plane array is populated with a mosaic of four thinned Lockheed $4096 \times 4096$ pixel CCDs. The camera provides a plate scale of 0.45 arcsec pixel $^{-1}$ and a total field of view of $1.16 \mathrm{deg} \times$ $1.16 \mathrm{deg}$. The $R$ images' total exposure time was $1800 \mathrm{~s}$, while the $\mathrm{H} \alpha+[\mathrm{N}$ II $]$ images totaled $5400 \mathrm{~s}$. Follow-up imagery in the same filters was obtained with the Mosaic CCD camera at the prime focus of the Kitt Peak National Observatory Mayall $4 \mathrm{~m}$ telescope. The Mosaic camera on the $4 \mathrm{~m}$ telescope has eight $2048 \times 4096$ SITe thinned CCDs, and an image scale of 0.26 arcsec pixel $^{-1}$. Imaging was carried out on the nights of 2010 February 7 and 9, and conditions were generally clear. Eighteen $R$-band images, each of $180 \mathrm{~s}$ duration (3240 s total exposure) and $18 \mathrm{H} \alpha+[\mathrm{N} \mathrm{II}]$ images of $1800 \mathrm{~s}$ each $(32,400 \mathrm{~s}$ exposure) were obtained. Images were dithered over both nights during each epoch.

After flat fielding and de-biasing, stand-alone Daophot (Stetson 1987) was used to align the images on each chip; then all of the chips were matched together. All of the continuum (hereafter " $R$ ") and all of the narrowband (hereafter " $[\mathrm{N}$ II]") images of each epoch were combined to create the deepest possible image. The images were stitched together using montage2, a mosaicking program within the stand-alone Daophot. After this process was completed individually for both the $[\mathrm{N} \mathrm{II}]$ and $R$-band images, the narrow- and broadband images were matched up with Daophot (which uses triangular stellar patterns for its matching algorithm).

Spectra of the brightest knots in the AT Cnc ejecta were obtained with the R-C spectrograph of the Kitt Peak National Observatory Mayall $4 \mathrm{~m}$ telescope. A $1581 \mathrm{~mm}^{-1}$ grating was used because of the faintness of the nebulosity. We combined, using IRAF imcombine, four images $(1 \times 600 \mathrm{~s}$ plus $3 \times 1800 \mathrm{~s}$, for total of $6000 \mathrm{~s}$ ) for the first slit position and two images
$(1800 \mathrm{~s} \times 2$, for a total of $3600 \mathrm{~s})$ for the second slit position. We extracted spectra of each knot using the IRAF task apall.

Ultraviolet imagery was also obtained with the NASA GALEX satellite. The GALEX image data include far-UV (FUV; $\lambda_{\text {eff }}=$ $1516 \AA, \Delta \lambda=256 \AA$ ) and near-UV (NUV; $\lambda_{\text {eff }}=2267 \AA, \Delta \lambda=$ $730 \AA$ ) images in circular fields of diameter 1.2 . The total exposure in the FUV filter is $1600 \mathrm{~s}$ while that in the NUV filter is $12,100 \mathrm{~s}$. The spatial resolution is $\sim 5^{\prime \prime}$. Details of the GALEX instrument and data characteristics can be found in Martin et al. (2005) and Morrissey et al. (2005). The imaging data have been processed under the standard GALEX survey pipeline.

\section{IMAGING OF THE AT Cnc SHELL}

The resulting $R$ and net $\mathrm{H} \alpha+[\mathrm{NII}$ (narrowband minus $R$ ) images from the KPNO $4 \mathrm{~m}$ telescope, taken in 2010 are shown in Figures 1 and 2, respectively. At $\mathrm{Cnc}$ is circled in both images. This is one of the deepest narrowband-broadband image pairs ever taken of any nova ejecta. The net narrowband image is dominated by the striking arcs running from the NE through $\mathrm{N}$, $\mathrm{W}$, and $\mathrm{S}$ of the central star. The two arcs, and their geometry are reminiscent of the hourglass-shaped nebulae of the LMC supernova SN87A (Lawrence et al. 2000) and the planetary nebula MyCn 18 (Sahai et al. 1999). The near coincidence of the two rings suggests that we are viewing the hourglass almost along its long symmetry axis. Both the rings are extremely fragmented, like the central ring of SN87A seen a decade and more after the supernova eruption. This morphology is suggestive of a fast wind colliding with slow or stationary ejecta from previous outbursts. The shock-dominated spectrum of the brightest knots (next section) supports this interpretation.

\section{SPECTRUM OF THE AT Cnc EJECTA}

We placed the $4 \mathrm{~m}$ spectrograph slit at two orientations and positions in order to get spectra of the two brightest knots in AT Cnc's shell. The first slit, at an angle of $6.6 \mathrm{deg}$ from north, spans two bright knots very close to each other. The second slit, at an angle of 119.9 deg from north, spans a single bright knot. The slit positions can be seen overlaid on the image of AT Cnc and its shell in Figure 2. Only the first (6000 s spectrum) yielded sufficient signal to clearly identify the primary emission lines. That spectrum is shown in Figure 3, which is dominated by the emission lines of [N $\mathrm{II}]$, [O III], and [O II]

The presence of the $[\mathrm{O}$ II] places an upper limit on the density of the emitting gas of about $3000 \mathrm{~cm}^{-3}$ (Appenzeller \& Oestreicher 1988). Unfortunately, our spectral resolution is too low to resolve the [O II] doublet and further constrain the density. As we show in Section 6, AT Cnc's luminosity (roughly $1 L_{\odot}$ ) and effective temperature (about $10 \mathrm{kK}$ ) are too low to photoionize the arcs of ejecta seen in Figure 2. The lack of Balmer lines and the presence of strong [N II] lines (see Figure 3 ) suggests a shock temperature in excess of $20 \mathrm{kK}$. The emission lines and their ratios are reminiscent of the spectra of the ejecta of the classical nova GK Per (nova 1901; Shara et al. 2012b) and the recurrent nova T Pyx (Contini \& Prialnik 1997). Both these are shock ionized due to the collision of rapidly outflowing ejecta with slower moving matter.

\section{GALEX UV IMAGERY}

In Figure 4, we compare the optical narrowband [N II] image with the GALEX satellite's stretched individual FUV and NUV images of AT Cnc. The small, faint "halo" seen surrounding 


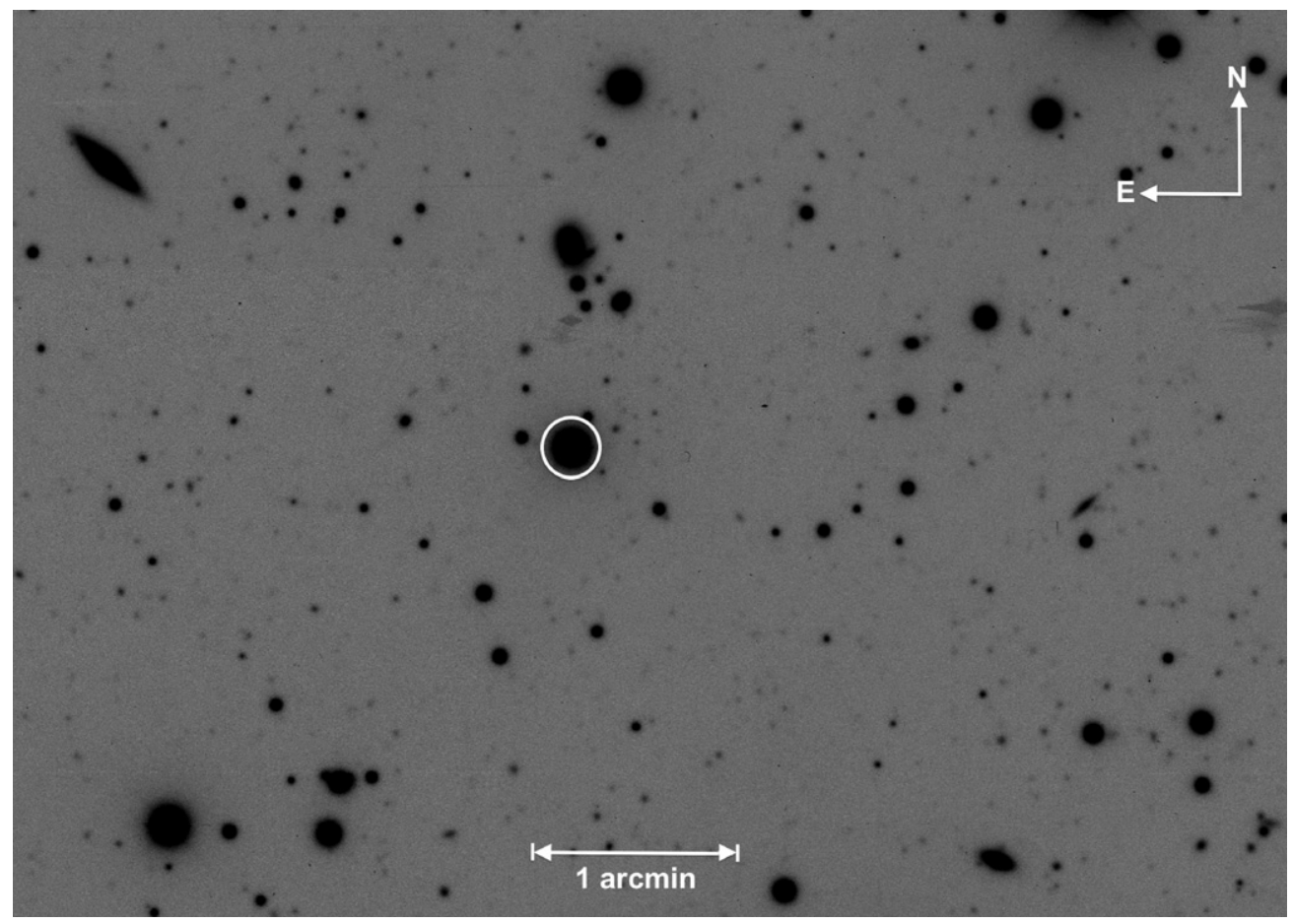

Figure 1. Deep R-band image of the field surrounding the Z Cam-type dwarf nova AT Cnc. AT Cnc is circled.

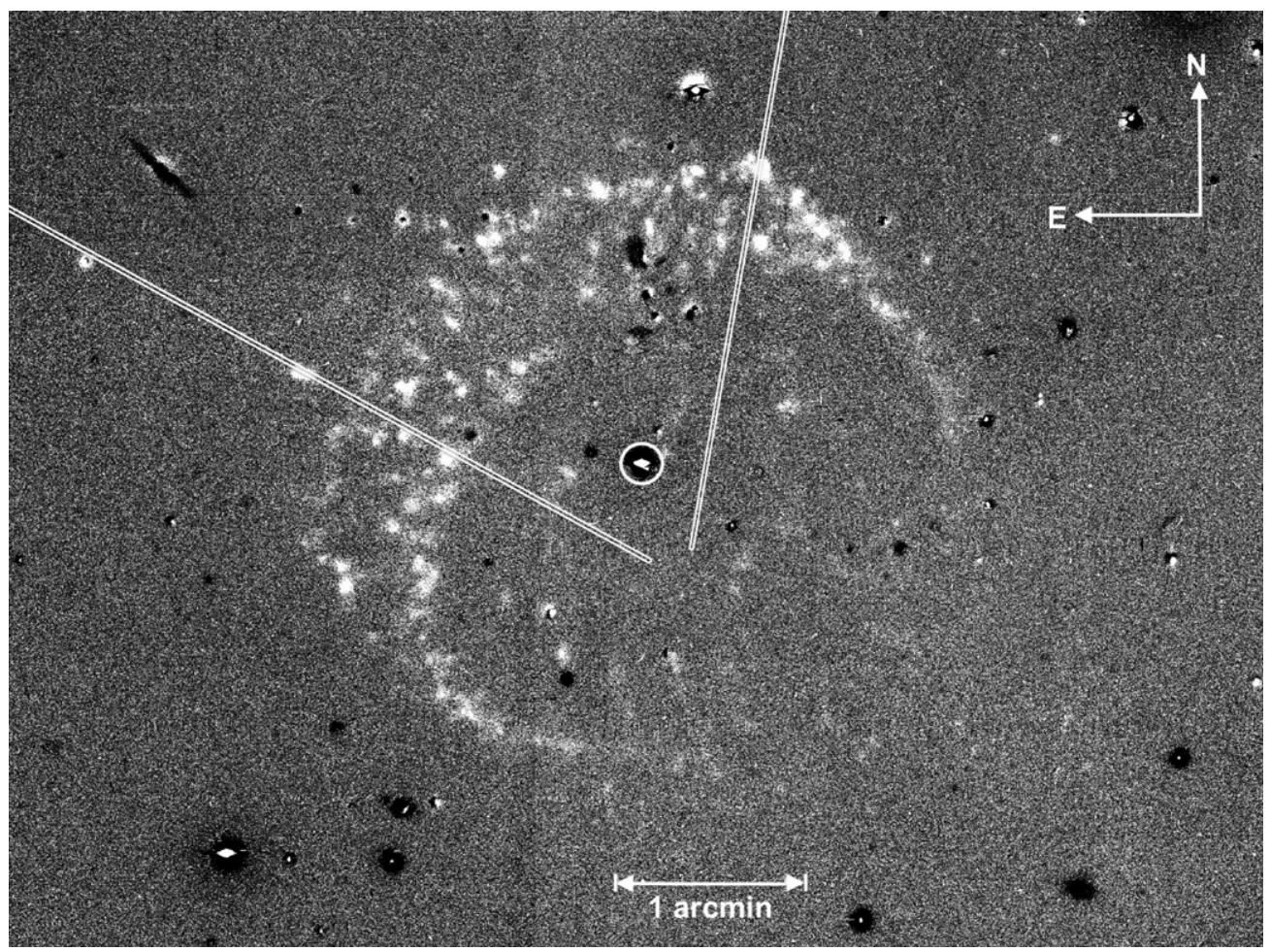

Figure 2. Net $\mathrm{H} \alpha+[\mathrm{N}$ II] image of AT Cnc. AT Cnc is circled. The positions of two slits used to obtain spectra of the AT Cnc ejecta are superposed on the image.

AT Cnc in the NUV image is instrumental (and seen surrounding other stars of similar brightness), but the extended FUV emission is not. The correspondence of the FUV emission to the 3 arcmin diameter [N II]-emitting shells is one-to-one.

\section{THE DISTANCE TO AC Cnc AND ITS EJECTED MASS}

Knowing the current size of the AT Cnc ejecta and the range of observed ejection velocities for novae allows us to set upper and lower limits to the time since AT Cnc's last nova eruption. Translating the angular size ( $3 \mathrm{arcmin}$ in diameter) into a linear size requires knowing the distance to AT Cnc. There is no published parallax or spectroscopic distance for AT Cnc, so we use the infrared period-luminosity-color (PLC) relation derived by Ak et al. (2007). This, in turn, demands a knowledge of the reddening to the system. Bruch \& Engel (1994) note that $E(B-V)=0.0$ for AC Cnc, YZ Cnc, and SY Cnc, all of which are located close to Galactic latitude $b=+30 \mathrm{deg}$. 


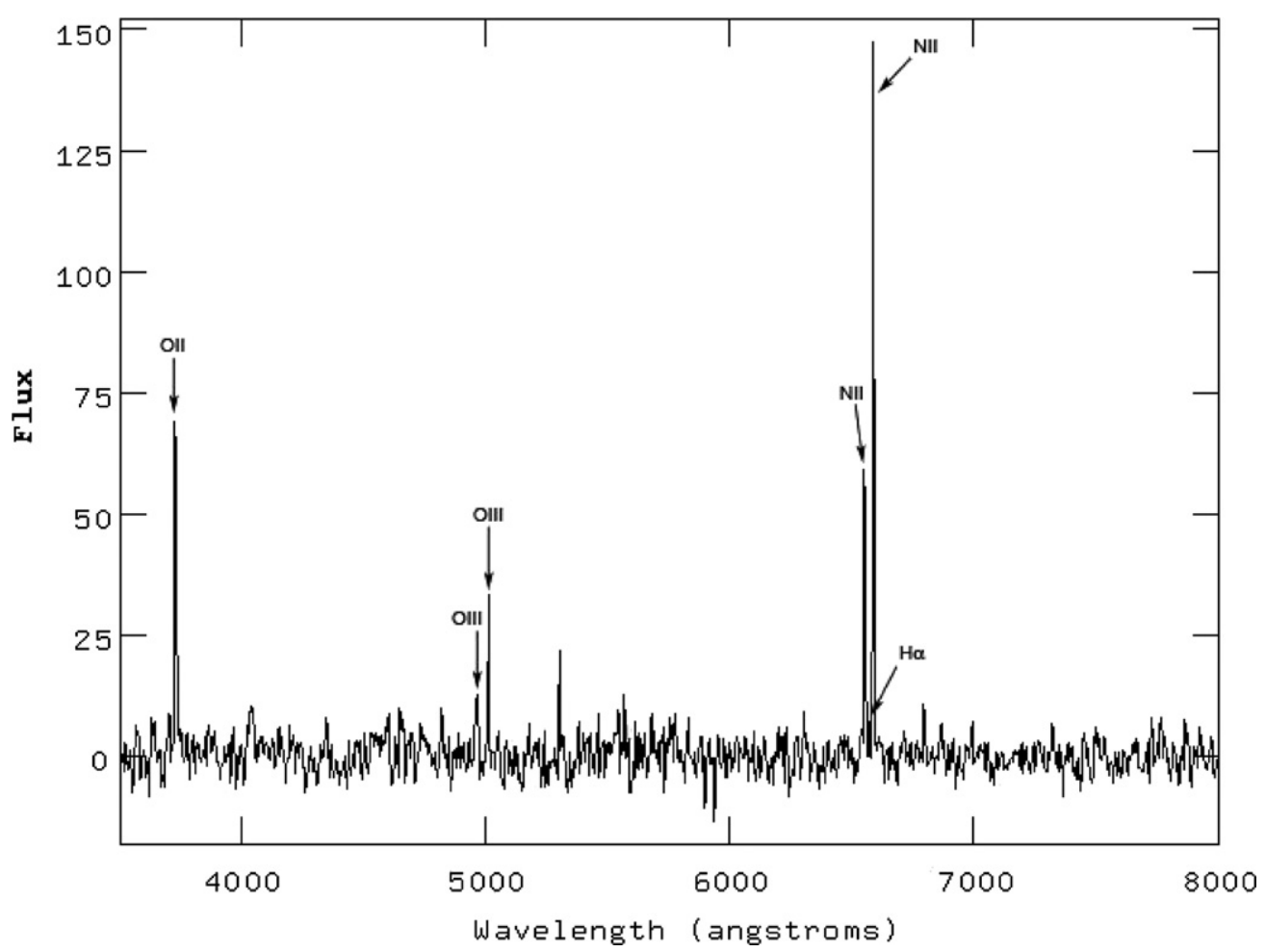

Figure 3. Optical spectrum of the brightest knot in the ejecta of AT Cnc.

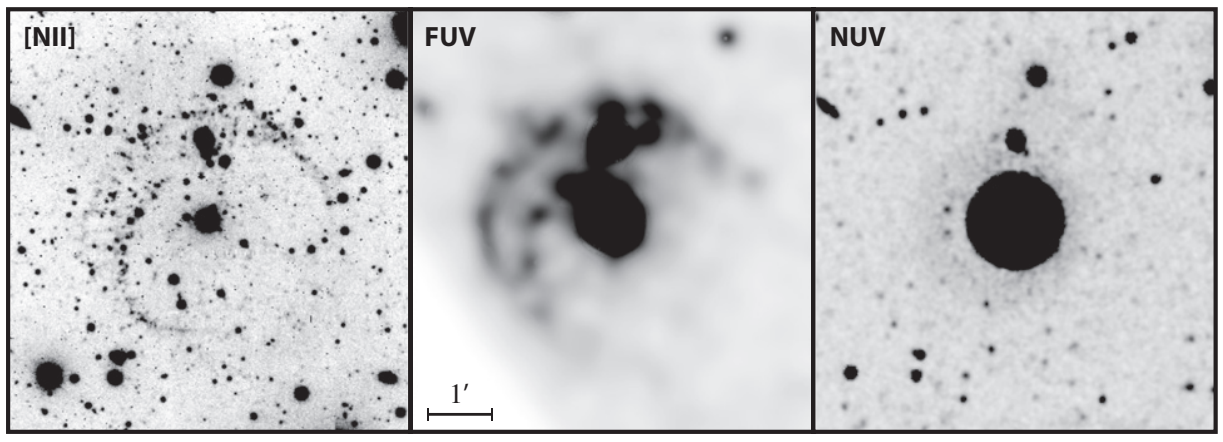

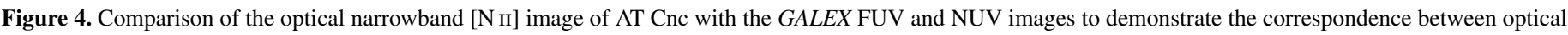
narrowband [N II] and FUV emission. The "halo" surrounding AT Cnc in the NUV image is instrumental.

We adopt the same value for AT Cnc (at $b=+31 \mathrm{deg}$ ). The distance predicted by the Ak et al. (2007) PLC method for AT $\mathrm{Cnc}$ is then $460 \mathrm{pc}$. The 1.5 arcmin radius of the AT Cnc ejecta corresponds to $0.2 \mathrm{pc}$ at that distance, and the system luminosity at maximum brightness is thus $\sim L_{\odot}$. The spectrum of AT Cnc is dominated by the Balmer lines of the accretion disk; He I is quite weak, and no trace is seen of He II. (The modest effective temperature and luminosity of AT Cnc are the basis of our claim in Section 4 above that the luminous ejecta of AT Cnc cannot be photoionized by the central star.)

We can place a very rough upper limit on the ejecta mass as follows. Roughly 100 "blobs" are seen in the emitting rings of Figure 2. Most of these blobs are unresolved, so we adopt an upper size limit of 1 arcsec for each one. This corresponds to a physical diameter of $\sim 7 \times 10^{13} \mathrm{~m}$. Assuming that each blob is spherical with a density less than $3000 \mathrm{~cm}^{-3}$ yields an upper limit to the mass in blobs of $\sim 5 \times 10^{-5} M_{\odot}$, in excellent agreement with theoretical predictions of nova ejecta masses (Yaron et al. 2005).

\section{WHEN DID AT Cnc LAST ERUPT AS A NOVA?}

Neither the encyclopedic summary of pre-telescopic transient stars of Ho (1962) nor the carefully culled list of Far Eastern observations of classical novae before 1800 AD of Stephenson (1987) contains a classical nova candidate close to the position of AT Cnc. This is in contrast to Z Cam, the oldest classical nova ever recovered. The dynamics of Z Cam's ejecta constrain its last eruption to have occurred more than 1300 years ago (Shara et al. 2012a), and Z Cam's position is consistent with that of the Chinese nova of 77 BCE (Johansson 2007).

Virtually all classical novae (with the exception of the 10 known Galactic recurrent novae) exhibit ejection velocities in the range 300-3000 $\mathrm{km} \mathrm{s}^{-1}$ (cf. Warner 1995, Table 5.2). Traveling at $3000(300) \mathrm{km} \mathrm{s}^{-1}$, with no deceleration, the AT Cnc ejecta could have reached their present size in no less (no more) than 63 (630) yr.

The ejecta of novae suffer significant deceleration on a timescale of 50-100 years, as suggested by Oort (1946) and 
directly measured by Duerbeck (1987). The observed expansion velocity dropped to half its initial value in $65,58,117$, and 67 years, respectively, for the four classical novae V603 Aql, GK Per, V476 Cyg, and DQ Her (Duerbeck 1987). The observed peak ejection velocities of these four novae were 1700, 1200, 725 , and $325 \mathrm{~km} \mathrm{~s}^{-1}$, respectively. The remarkably small range in deceleration half-lifetimes $t$ for the four novae noted above are in excellent accord with the Oort snow-plough model's predictions. They demonstrate that AT Cnc's ejecta must have undergone at least one deceleration half-lifetime since its eruption. Thus, the time since AT Cnc's last nova eruption is almost certainly double the lower limit noted above (i.e., 126 years) or greater. We cannot more strongly constrain the upper limit other than to say it is of the order of 1000 years.

As a "sanity check" we note that GK Per (which erupted 111 years ago) is at the same distance as AT Cnc, and that it displays a shell almost half the size (Shara et al. 2012b) of the AT Cnc ejecta. GK Per will achieve AT Cnc's angular size in about two centuries, supporting our simple estimates of an age of a few centuries for AT Cnc.

The expansion rate of AT Cnc's ejecta should be directly measurable within a decade, providing a better-determined lower limit to its age. Measuring the deceleration will take longer, but its determination will enable the best possible estimate of the time elapsed since AT Cnc last erupted as a nova. This will, in turn, provide us with the first quantitatively measured estimate of the time required for an old nova to revert to its subsequent dwarf nova phase.

\section{IMPLICATIONS OF THE EXISTENCE OF THE AT Cnc SHELL}

Our detection of a second nova shell surrounding a Z Camtype dwarf nova further supports the claim that these stars are intimately connected with nova eruptions. There are 44 known and suspected Z Cam-type stars as of 2012 April; we have surveyed only half of them. An up-to-date list is maintained by Ringwald at https://sites.google.com/site/thezcamlist/the-list. If $\mathrm{Z}$ Cam were the only dwarf nova with a detected nova shell then one could have argued that we fortuitously captured a very rare and transient event. The existence of the AT Cnc old nova ejecta argues against that interpretation. We will soon announce a third Z Cam star surrounded by ejecta, further supporting our claim that old novae and Z Cam stars are intimately connected: strong evidence supporting hibernation.

\section{SUMMARY AND CONCLUSIONS}

We report the optical narrowband detection of fragmented rings, 3 arcmin in diameter, surrounding the dwarf nova AT
Cancri. The shell geometry is suggestive of bipolar, conical ejection seen nearly pole-on. A spectrum of the brightest part of the AT Cnc ejecta is similar to that of the ejecta of the classical nova GK Per, and of $\mathrm{Z}$ Cam, dominated by [N II], [O II], and [O III] emission. The ejecta must be shock ionized. GALEX FUV imagery reveals a similar-sized, FUV-emitting shell. We determine that AT Cnc is about 460 pc from Earth, with a system luminosity at maximum brightness that is $\sim L_{\odot}$. The 1.5 arcmin radius of the AT Cnc ejecta corresponds to $0.2 \mathrm{pc}$ at that distance, with a maximum shell mass of $\sim 5 \times 10^{-5} M_{\odot}$, in excellent agreement with theoretical predictions of nova ejecta masses.

Galaxy Evolution Explorer (GALEX) is a NASA Small Explorer, launched in 2003 April. We gratefully acknowledge NASA's support for construction, operation, and science analysis for the GALEX mission.

M.M.S. gratefully acknowledges helpful conversations about AT Cnc and dwarf nova shells with Howard Bond and Christian Knigge.

\section{REFERENCES}

Ak, T., Bilir, S., Ak, S., \& Retter, A. 2007, New Astron., 12, 446

Appenzeller, I., \& Oestreicher, R. 1988, AJ, 95, 45

Bond, H. E., \& Tifft, W. G. 1974, PASP, 86, 981

Bruch, A., \& Engel, A. 1994, A\&AS, 104, 79

Collazzi, A. C., Schaefer, B. E., Xiao, L., et al. 2009, AJ, 138, 1846

Contini, M., \& Prialnik, D. 1997, ApJ, 475, 803

Duerbeck, H. W. 1987, Ap\&SS, 131, 461

Ho, P. Y. 1962, Vistas Astron., 5, 127

Johansson, G. H. I. 2007, Nature, 448, 251

Lawrence, S. S., Sugerman, B. E., Bouchet, P., et al. 2000, ApJ, 537, L123

Martin, D. C., Fanson, J., Schiminovich, D., et al. 2005, ApJ, 619, L1

Morrissey, P., Schiminovich, D., Barlow, T. A., et al. 2005, ApJ, 619, L7

Nogami, D., Masuda, S., Kato, T., \& Hirata, R. 1999, PASJ, 51, 115

Oort, J. H. 1946, MNRAS, 106, 159

Osaki, Y. 1974, PASJ, 26, 429

Robinson, E. L. 1975, AJ, 80, 515

Sahai, R., Dayal, A., Watson, A. M., et al. 1999, AJ, 118, 468

Shara, M., Livio, M., Moffat, A. F. J., \& Orio, M. 1986, ApJ, 311, 163

Shara, M. M. 1989, PASP, 101, 5

Shara, M. M., Martin, C. D., Seibert, M., et al. 2007, Nature, 446, 159

Shara, M. M., Mizusawa, T., Zurek, D., et al. 2012a, ApJ, 756, 107

Shara, M. M., Zurek, D., De Marco, O., et al. 2012b, AJ, 143, 143

Stephenson, F. R. 1987, in RS Ophiuchi (1985) and the Recurrent Nova Phenomenon, ed. M. F. Bode (Utrecht: VNU Science), 105

Stetson, P. B. 1987, PASP, 99, 191

Warner, B. 1995, Cataclysmic Variable Stars (Cambridge: Cambridge Univ. Press)

Williams, G. G., Olszewski, E., Lesser, M. P., \& Burge, J. H. 2004, Proc. SPIE, 5492, 787

Yaron, O., Prialnik, D., Shara, M. M., \& Kovetz, A. 2005, ApJ, 623, 398 